TUHEP-TH-02139

\title{
Muon anomalous magnetic moment and lepton flavor violation in MSSM
}

\author{
Xiao-Jun Bi.* Yu-ping Kuang ${ }^{\dagger}$ and Yong-Hong An \\ Department of Physics, Tsinghua University, \\ Beijing 100084, People's Republic of China
}

(Dated: May 11, 2019)

\begin{abstract}
We give a thorough analysis of the correlation between the muon anomalous magnetic moment and the radiative lepton flavor violating (LFV) processes within the minimal supersymmetric standard model. We find that in the case when the slepton mass eigenstates are nearly degenerate, $\delta a_{\mu}$, coming from SUSY contributions, hardly depends on the lepton flavor mixing and, thus, there is no direct relation between $\delta a_{\mu}$ and the LFV processes. On the contrary, if the first two generations' sleptons are much heavier than the 3rd one, i.e., in the effective SUSY scenario, the two quantities are closely related. In the latter scenario, the SUSY parameter space to account for the experimental $\delta a_{\mu}$ is quite different from the case of no lepton flavor mixing. Especially, the Higgsino mass parameter $\mu$ can be either positive or negative.
\end{abstract}

*Email: bixj@mail.tsinghua.edu.cn

$\dagger$ Email: ypkuang@mail.tsinghua.edu.cn 


\section{INTRODUCTION}

Recently, the Brookhaven E821 Collaboration announced their new experimental result on muon anomalous magnetic moment, $a_{\mu}=\left(g_{\mu}-2\right) / 2$, with improved statistics[1], which is twice precision of their 2001 result [2]. The present discrepancy between the standard model (SM) prediction and the measurement, depending on the SM hadronic corrections to $a_{\mu}$, is

$$
\begin{aligned}
& a_{\mu}^{e x p}-a_{\mu}^{S M}=26(10) \times 10^{-10} \\
& \text { or } 17(11) \times 10^{-10}
\end{aligned}
$$

lying between $1.6 \sigma$ and $2.6 \sigma$.

Since the first announcement of existing discrepancy between theory and experiment on $a_{\mu}$, there appeared a lot of works on this subject trying to explain the result in various extensions of the SM, among which the most promising new physics is the minimal supersymmetric standard model (MSSM) 3]. Although the present E821's measurement can not provide compelling evidence in favor of new physics, it is generally expected that this deviation will be confirmed when both the experimental and theoretical errors are reduced and this result can now be used to put constraint on the supersymmetric (SUSY) parameters.

The extensive studies show that it is easy to accommodate $\delta a_{\mu} \sim(10 \sim 70) \times 10^{-10}$ within the MSSM framework if the SUSY particles are as 'light' as about a few hundred GeV $[3]$. The positive sign of the Higgsino mass parameter, $\mu$, is strongly favored by the present value of $\delta a_{\mu}$. Since in most parameter space the main SUSY contribution to $a_{\mu}$ comes from exchanging chargino and scalar muon neutrino virtual particles, which is approximately proportional to $\mu M_{2} \tan \beta$, the $\operatorname{sign}$ of $\mu$ is thus positive relative to $M_{2}$, the wino mass parameter, provided that SUSY helps to enhance $a_{\mu}$.

In this work we will study the SUSY contributions to $a_{\mu}$ in the case when considering the lepton flavor mixing in the soft breaking sector. Different from the similar numerical studies of lepton flavor mixing effects on $a_{\mu}[4]$, we will give a thorough analysis of the correlation between the SUSY contributions to $a_{\mu}$ and to lepton flavor violation (LFV). We find in the case that the sleptons and sneutrinos are nearly degenerate, $\delta a_{\mu}$ has no direct relation with the LFV processes. Actually, this is the usual case which has been extensively studied in the literature [3]. However, it is most important to take into account the effects of lepton flavor

mixing in the case of effective SUSY scenario, where $\delta a_{\mu}$ can only arise when the slepton 


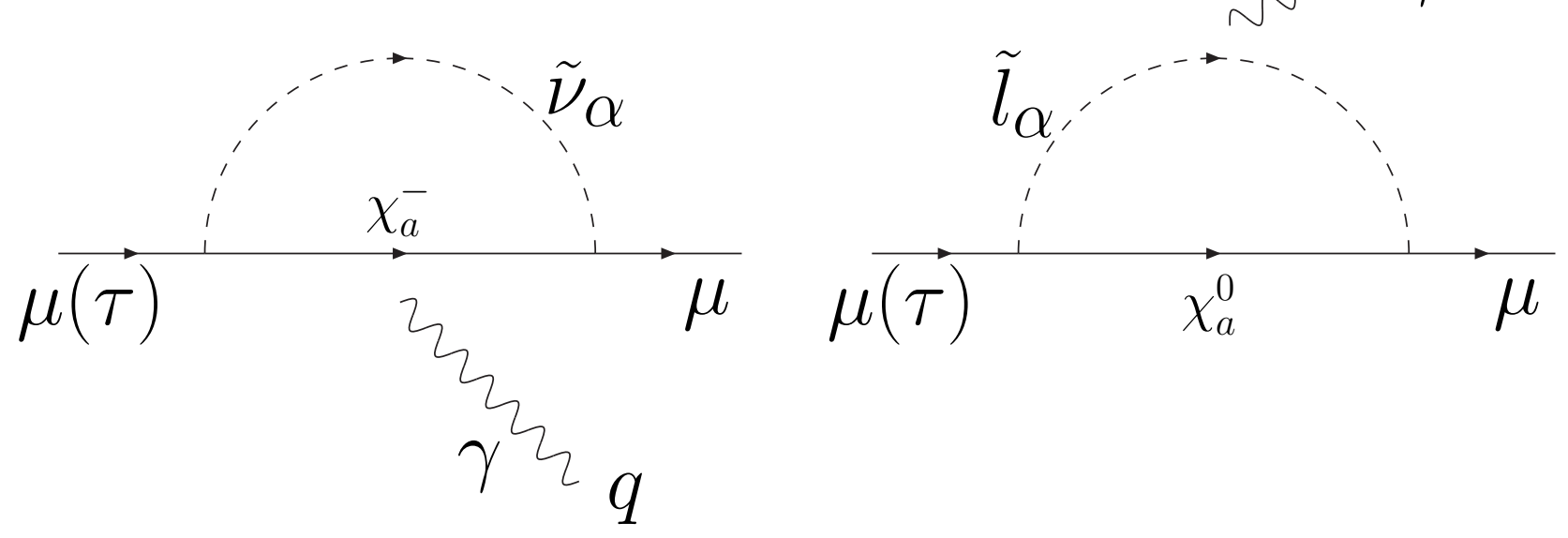

FIG. 1: Feynman diagrams of the one-loop SUSY contribution to $a_{\mu}$ (and the process $\tau \rightarrow \mu \gamma$ ) via the exchange of a chargino (left) and via a neutralino (right).

mixing between the second and the third generations is introduced.

We find that in the effective SUSY scenario, the SUSY parameter space may be quite different from those without considering the slepton mixing. The sign of $\mu$ can be either positive or negative, to enhance $a_{\mu}$, depending on the lepton flavor mixing angles. Small $\tan \beta$ is more favored in this case.

The paper is organized as follows. In the next section we give the analytic expressions for the SUSY contributions to $a_{\mu}$ and the branching ratio of LFV processes $l_{i} \rightarrow l_{j} \gamma$. In section III, we will present the numerical results and some approximate upper bound on $\delta a_{\mu}$. Finally, we give summary and conclusions in section IV.

\section{ANALYTIC EXPRESSIONS}

\section{A. Expressions of $\delta a_{\mu}$ and $\operatorname{Br}\left(l_{i} \rightarrow l_{j} \gamma\right)$ in MSSM}

Since the muon's anomalous magnetic moment and the LFV processes $l_{i} \rightarrow l_{j} \gamma$ arise from similar operators, the effective Lagrangian related to $a_{\mu}$ and radiative LFV process can be 
written in one form,

$$
\mathcal{L}_{e f f}=e \frac{m_{i}}{2} \bar{l}_{j} \sigma_{\alpha \beta} F^{\alpha \beta}\left(A_{L}^{i j} P_{L}+A_{R}^{i j} P_{R}\right) l_{i},
$$

where $P_{L, R}=\frac{1}{2}\left(1 \mp \gamma_{5}\right)$ are the chirality projection operators and $i(j)$ denotes the initial (final) lepton flavor. The muon anomalous magnetic moment is given by

$$
a_{\mu}=m_{\mu}^{2}\left(A_{L}^{22}+A_{R}^{22}\right)
$$

while the branching ratio of $l_{i} \rightarrow l_{j} \gamma$ is given by

$$
B R\left(l_{i} \rightarrow l_{j} \gamma\right)=\frac{\alpha_{e m}}{4} m_{i}^{5}\left(\left|A_{L}^{i j}\right|^{2}+\left|A_{R}^{i j}\right|^{2}\right) / \Gamma_{i}
$$

with $\Gamma_{i}[\underline{5}]$ being the width of $l_{i}$.

The SUSY contribution to the form factors $A_{L}$ and $A_{R}$ is given by the photon-penguin diagrams via exchanging (i) chargino-sneutrino and (ii) neutralino-slepton, as shown in FIG. 1. The analytic expressions for $\delta a_{\mu}$ from the neutralino and chargino exchange are

$$
\begin{aligned}
\delta a_{\mu}^{(n)}= & -\frac{1}{32 \pi^{2}} \frac{e^{2}}{\cos ^{2} \theta_{W}} \frac{m_{\mu}^{2}}{m_{\tilde{l}_{\alpha}}^{2}} . \\
& {\left[\left(A^{i \alpha a^{*}} A^{i \alpha a}+B^{i \alpha a^{*}} B^{i \alpha a}\right) F_{1}\left(k_{\alpha a}\right)+\frac{m_{\chi_{a}^{0}}}{m_{\mu}} \operatorname{Re}\left(A^{i \alpha a^{*}} B^{i \alpha a}\right) F_{2}\left(k_{\alpha a}\right)\right] }
\end{aligned}
$$

and

$$
\begin{aligned}
\delta a_{\mu}^{(c)}= & \frac{g_{2}^{2}}{16 \pi^{2}} \frac{m_{\mu}^{2}}{m_{\tilde{\nu}_{\alpha}}^{2}} Z_{\tilde{\nu}}^{i \alpha} Z_{\tilde{\nu}}^{i \alpha^{*}} . \\
& {\left[\left(Z_{1 a}^{+*} Z_{1 a}^{+}+\frac{m_{\mu}^{2}}{2 M_{W}^{2} \cos ^{2} \beta} Z_{2 a}^{-*} Z_{2 a}^{-}\right) F_{3}\left(k_{\alpha a}\right)+\frac{m_{\chi_{a}^{-}}}{\sqrt{2} M_{W} \cos \beta} \operatorname{Re}\left(Z_{1 a}^{+} Z_{2 a}^{-}\right) F_{4}\left(k_{\alpha a}\right)\right], }
\end{aligned}
$$

respectively with index $i=2$. In the above expressions the $A$ and $B$ are the lepton-sleptonneutralino coupling vertices given by

$$
\begin{aligned}
A^{i \alpha a} & =\left(Z_{\tilde{L}}^{i \alpha}\left(Z_{N}^{1 a}+Z_{N}^{2 a} \cot \theta_{W}\right)-\cot \theta_{W} \frac{m_{i}}{M_{W} \cos \beta} Z_{\tilde{L}}^{(i+3) \alpha} Z_{N}^{3 a}\right) \\
B^{i \alpha a} & =-\left(2 Z_{\tilde{L}}^{(i+3) \alpha} Z_{N}^{1 a^{*}}+\cot \theta_{W} \frac{m_{i}}{M_{W} \cos \beta} Z_{\tilde{L}}^{i \alpha} Z_{N}^{3 a *}\right)
\end{aligned}
$$

where $Z_{\tilde{L}}$ is the $6 \times 6$ slepton mixing matrix and $Z_{N}$ is the neutralino mixing matrix. Similarly, $Z_{\tilde{\nu}}$ is the sneutrino mixing matrix, while $Z^{+}$and $Z^{-}$are the mixing matrices for the charginos. The definitions of these mixing matrices and the expressions of $F_{i}$ 's are given in the appendix. 
For the processes $l_{i} \rightarrow l_{j} \gamma$, the contribution from neutralino exchange gives

$$
\begin{aligned}
& A_{L}^{i j(n)}=-\frac{1}{32 \pi^{2}}\left(\frac{e}{\sqrt{2} \cos \theta_{W}}\right)^{2} \frac{1}{m_{\tilde{l}_{\alpha}}^{2}}\left[B^{j \alpha a^{*}} B^{i \alpha a} F_{1}\left(k_{\alpha a}\right)+\frac{m_{\chi_{a}^{0}}}{m_{i}} B^{j \alpha a^{*}} A^{i \alpha a} F_{2}\left(k_{\alpha a}\right)\right], \\
& A_{R}^{i j(n)}=A_{L}^{(n)}(B \leftrightarrow A),
\end{aligned}
$$

while the corresponding contribution coming from chargino exchange is

$$
\begin{aligned}
A_{L}^{i j(c)}= & \frac{g_{2}^{2}}{32 \pi^{2}} Z_{\tilde{\nu}}^{i \alpha^{*}} Z_{\tilde{\nu}}^{j \alpha} \frac{1}{m_{\tilde{\nu}_{\alpha}}^{2}}\left[Z_{2 a}^{-} Z_{2 a}^{-*} \frac{m_{i} m_{j}}{2 M_{W}^{2} \cos ^{2} \beta} F_{3}\left(k_{\alpha a}\right)\right. \\
& \left.+\frac{m_{\chi_{a}^{-}}}{\sqrt{2} M_{W} \cos \beta} Z_{1 a}^{+} Z_{2 a}^{-} \frac{m_{j}}{m_{i}} F_{4}\left(k_{\alpha a}\right)\right], \\
A_{R}^{i j(c)}= & \frac{g_{2}^{2}}{32 \pi^{2}} Z_{\tilde{\nu}}^{i \alpha^{*}} Z_{\tilde{\nu}}^{j \alpha} \frac{1}{m_{\tilde{\nu}_{\alpha}}^{2}}\left[Z_{1 a}^{+} Z_{1 a}^{+*} F_{3}\left(k_{\alpha a}\right)+\frac{m_{\chi_{a}^{-}}}{\sqrt{2} M_{W} \cos \beta} Z_{1 a}^{+*} Z_{2 a}^{-*} F_{4}\left(k_{\alpha a}\right)\right] .
\end{aligned}
$$

\section{B. Flavor structure on the interaction basis}

The expressions for $\delta a_{\mu}$ and $\operatorname{Br}\left(l_{i} \rightarrow l_{j} \gamma\right)$ in the last subsection show that there is close relations between the two quantities. We notice that all these expressions are given in the mass eigenstates of the SUSY particles and the lepton flavor mixing is presented in the mixing matrices on the interaction vertices. These expressions are suitable for numerical calculations. However, to analyze the flavor structure of the amplitude, it is more convenient to work on the interaction basis, which is defined as the basis where the lepton mass matrix and the gauge coupling vertices are all diagonal. On this basis there are much more Feynman diagrams than those in FIG. 1]. For example, the vertex $A$ given in Eq. (8) actually contains three different interaction vertices, $l_{i}^{L}-\tilde{l}_{i}^{L}-\tilde{B}, l_{i}^{L}-\tilde{l}_{i}^{L}-\tilde{W}$, and $l_{i}^{L}-\tilde{l}_{i}^{R}-\tilde{H}_{D}$ on this basis. Thus only the $A^{*} A$ term in Eq. (6) represents 9 different Feynman diagrams.

On this basis the slepton and sneutrino mass matrices are generally not diagonal. We first give the form of these mass matrices. The slepton mass matrix can be written in a general form as

$$
M_{\tilde{l}}^{2}=\left(\begin{array}{cc}
Z_{L} m_{L}^{2} Z_{L}^{\dagger} & -m_{l}\left(\mu \tan \beta+A_{l}^{*}\right) \\
-m_{l}\left(\mu^{*} \tan \beta+A_{l}\right) & Z_{R} m_{R}^{2} Z_{R}^{\dagger}
\end{array}\right)
$$

where

$$
\begin{aligned}
& m_{L}^{2}=m_{\tilde{l}}^{2}+m_{l}^{2}+\cos 2 \beta\left(-\frac{1}{2}+\sin ^{2} \theta_{W}\right) M_{Z}^{2} \\
& m_{R}^{2}=m_{\tilde{r}}^{2}+m_{l}^{2}-\cos 2 \beta \sin ^{2} \theta_{W} M_{Z}^{2}
\end{aligned}
$$


and $m_{l}$ is the diagonal mass matrix of leptons. Here $m_{\tilde{l}}^{2}$ and $m_{\tilde{r}}^{2}$ are diagonal matrices with their diagonal elements representing the mass squares of $\left(\tilde{e}_{L}, \tilde{\mu}_{L}, \tilde{\tau}_{L}\right)$ and $\left(\tilde{e}_{R}, \tilde{\mu}_{R}, \tilde{\tau}_{R}\right)$ respectively. $Z_{L}$ and $Z_{R}$ represent the mixing matrices in the left- and right-handed sleptons. In this work we consider the mixing between the second and the third generations (Thereafter we can completely ignore the first generation). $Z_{L}$ is then given by

$$
Z_{L}=\left(\begin{array}{cc}
c_{L} & s_{L} \\
-s_{L} & c_{L}
\end{array}\right), \text { with } c_{L}=\cos \theta_{L}, \quad s_{L}=\sin \theta_{L}
$$

while $m_{L}^{2}$ is given by

$$
m_{L}^{2}=\left(\begin{array}{cc}
m_{2}^{2} & \\
& m_{3}^{2}
\end{array}\right)
$$

On this basis the sneutrino mass matrix can be written as

$$
M_{\tilde{\nu}}^{2}=Z_{L} m_{\tilde{l}}^{2} Z_{L}^{\dagger}+\frac{1}{2} \cos 2 \beta M_{Z}^{2}
$$

Before giving the relations between $\delta a_{\mu}$ and $\operatorname{Br}(\tau \rightarrow \mu \gamma)$ in the next section, we first give an analysis of the flavor structure of the form factors $A_{L}$ and $A_{R}$ in the following. From Eqs. (10)-(13), we can see that the flavor structure of $A_{L(R)}$ is approximately proportional to $\left(M_{\tilde{\nu}}^{2}\right)_{i j}^{-1}$ or $\left(M_{\tilde{l}}^{2}\right)_{i j}^{-1}$, noticing that the functions $F_{i}\left(k_{\alpha a}\right)$ are quite flat in an appropriate range of $k_{\alpha a}$. The numerical results in the next section justifies our analysis given here ${ }^{1}$. It is easy to get, on the interaction basis,

$$
\left(M_{\tilde{\nu}}^{2}\right)^{-1}=\left(\begin{array}{cc}
\frac{c_{L}^{2}}{m_{2}^{2}}+\frac{s_{L}^{2}}{m_{3}^{2}} & c_{L} s_{L} \frac{m_{2}^{2}-m_{3}^{2}}{m_{2}^{2} m_{3}^{2}} \\
c_{L} s_{L} \frac{m_{2}^{2}-m_{3}^{2}}{m_{2}^{2} m_{3}^{2}} & \frac{s_{L}^{2}}{m_{2}^{2}}+\frac{c_{L}^{2}}{m_{3}^{2}}
\end{array}\right) .
$$

$\delta a_{\mu}$ is approximately proportional to the inverse mass square of $\tilde{\nu}_{\mu}-\tilde{\nu}_{\mu}$, which is noted as

$$
F\left(\tilde{\nu}_{\mu}-\tilde{\nu}_{\mu}\right)=\frac{c_{L}^{2}}{m_{2}^{2}}+\frac{s_{L}^{2}}{m_{3}^{2}}
$$

while $\tau \rightarrow \mu \gamma$ is approximately proportional to that of $\tilde{\nu}_{\tau}-\tilde{\nu}_{\mu}$, which is

$$
F\left(\tilde{\nu}_{\tau}-\tilde{\nu}_{\mu}\right)=\frac{1}{2} \sin 2 \theta_{L} \frac{m_{2}^{2}-m_{3}^{2}}{m_{2}^{2} m_{3}^{2}}
$$

\footnotetext{
${ }^{1}$ In other works, such as in Ref $[4]$, similar relations are given under the approximation that all the SUSY particles are degenerate.
} 
We consider the following two limit cases:

$$
F\left(\tilde{\nu}_{\mu}-\tilde{\nu}_{\mu}\right) \rightarrow \begin{cases}\frac{1}{m^{2}}, & \text { if } m_{2}^{2} \approx m_{3}^{2} \approx m^{2} \\ \frac{s_{L}^{2}}{m_{3}^{2}}, & \text { if } m_{2}^{2} \gg m_{3}^{2}\end{cases}
$$

while

$$
F\left(\tilde{\nu}_{\tau}-\tilde{\nu}_{\mu}\right) \rightarrow\left\{\begin{array}{l}
\frac{1}{2} \sin 2 \theta_{L} \frac{\Delta m^{2}}{m^{4}}, \quad \text { if } m_{2}^{2} \approx m_{3}^{2} \approx m^{2} \\
\frac{1}{2} \sin 2 \theta_{L} \frac{1}{m_{3}^{2}}, \quad \text { if } m_{2}^{2} \gg m_{3}^{2}
\end{array} .\right.
$$

In the first case with $m_{2}^{2} \approx m_{3}^{2}=m^{2}$, we can see that $\delta a_{\mu}$ does not depend on the mixing angle $\theta_{L}$ and has no direct relation with $\operatorname{Br}(\tau \rightarrow \mu \gamma)$. Thus models with gravity or gauge mediated supersymmetry breaking may predict that $\delta a_{\mu}$ has nothing to do with the mixing angle $\theta_{L}$, as already noticed in $\operatorname{Ref}[\underline{6}]$. Thus, to study $\delta a_{\mu}$ in the first case is actually equivalent to the case of no lepton flavor mixing in the soft sector, which has been extensively studied in the literature [3]. The second case leads us to the effective SUSY scenario[7], where the first two generations' sfermions are as heavy as about $20 \mathrm{TeV}$ while the 3rd generation's sfermions are kept in a few hundred $\mathrm{GeV}$. In this case $\delta a_{\mu}$ and $\operatorname{Br}(\tau \rightarrow \mu \gamma)$ are closely related; Increase $\theta_{L}$ to enhance $\delta a_{\mu}$ will unavoidably lead to large $\operatorname{Br}(\tau \rightarrow \mu \gamma)$. We have to consider the two quantities simultaneously and take the experimental bound on $\operatorname{Br}(\tau \rightarrow \mu \gamma)$ into account.

The inverse of the mass square of sleptons, on the interaction basis, is approximately given by

$$
\left(M_{\tilde{l}}^{2}\right)^{-1} \approx\left(\begin{array}{cc}
A & C \\
C^{\dagger} & B
\end{array}\right)
$$

with

$$
A \approx\left(M_{\tilde{\nu}}^{2}\right)^{-1}, \quad B \approx A\left(\theta_{L} \rightarrow \theta_{R}\right)
$$

and

$$
C \approx m_{\tau} \mu \tan \beta \frac{m_{2}^{2}-m_{3}^{2}}{m_{2}^{2} m_{3}^{2}} \cdot\left[\begin{array}{ll}
\frac{1}{4} \sin 2 \theta_{L} \sin 2 \theta_{R} \frac{m_{2}^{2}-m_{3}^{2}}{m_{2}^{2} m_{3}^{2}} & \frac{1}{2} \sin 2 \theta_{L}\left(\frac{s_{R}^{2}}{m_{2}^{2}}+\frac{c_{R}^{2}}{m_{3}^{2}}\right) \\
\frac{1}{2} \sin 2 \theta_{R}\left(\frac{s_{L}^{2}}{m_{2}^{2}}+\frac{c_{L}^{2}}{m_{3}^{2}}\right) & \left(\frac{s_{L}^{2}}{m_{2}^{2}}+\frac{c_{L}^{2}}{m_{3}^{2}}\right)\left(\frac{s_{R}^{2}}{m_{2}^{2}}+\frac{c_{R}^{2}}{m_{3}^{2}}\right)
\end{array}\right] .
$$

In matrix $C$ we have omitted the terms proportional to $m_{\mu}$. From the above expressions we know $F\left(\tilde{\mu}_{L}-\tilde{\mu}_{L}\right)$ is the same as that of $F\left(\tilde{\nu}_{\mu}-\tilde{\nu}_{\mu}\right)$, while $F\left(\tilde{\mu}_{R}-\tilde{\mu}_{R}\right)$ is gotten by changing $\theta_{L}$ to $\theta_{R}$ in $F\left(\tilde{\nu}_{\mu}-\tilde{\nu}_{\mu}\right)$. The most interesting result is that of $F\left(\tilde{\mu}_{L}-\tilde{\mu}_{R}\right)$, given by

$$
F\left(\tilde{\mu}_{L}-\tilde{\mu}_{R}\right)=\frac{1}{4} m_{\tau} \mu \tan \beta \sin 2 \theta_{L} \sin 2 \theta_{R}\left(\frac{m_{2}^{2}-m_{3}^{2}}{m_{2}^{2} m_{3}^{2}}\right)^{2} .
$$


The other two quantities related to $\tau \rightarrow \mu \gamma$ are

$$
F\left(\tilde{\mu}_{L}-\tilde{\tau}_{R}\right)=\frac{1}{2} m_{\tau} \mu \tan \beta \sin 2 \theta_{L}\left(\frac{m_{2}^{2}-m_{3}^{2}}{m_{2}^{2} m_{3}^{2}}\right)\left(\frac{s_{R}^{2}}{m_{2}^{2}}+\frac{c_{R}^{2}}{m_{3}^{2}}\right),
$$

and

$$
F\left(\tilde{\mu}_{R}-\tilde{\tau}_{L}\right)=\frac{1}{2} m_{\tau} \mu \tan \beta \sin 2 \theta_{R}\left(\frac{m_{2}^{2}-m_{3}^{2}}{m_{2}^{2} m_{3}^{2}}\right)\left(\frac{s_{L}^{2}}{m_{2}^{2}}+\frac{c_{L}^{2}}{m_{3}^{2}}\right)
$$

respectively.

Similarly, we consider the limit case of nearly degenerate sleptons, $m_{2}^{2} \approx m_{3}^{2} \approx m^{2}$. The other term, omitted in Eq. (28), which is proportional to $m_{\mu}$, may become important. Then we have

$$
F\left(\tilde{\mu}_{L}-\tilde{\mu}_{R}\right) \approx \frac{\mu \tan \beta}{m^{4}}\left[m_{\mu}+\frac{1}{4} m_{\tau} \sin 2 \theta_{L} \sin 2 \theta_{R}\left(\frac{\Delta m^{2}}{m^{2}}\right)^{2}\right] \approx \frac{\mu \tan \beta}{m^{4}} m_{\mu} .
$$

We can thus reach the same conclusion as before, that is, if $m_{2}^{2} \approx m_{3}^{2} \approx m^{2}, \delta a_{\mu}$ has no direct relation to slepton mixing angles. In case of $m_{2} \gg m_{3}$, we have

$$
F\left(\tilde{\mu}_{L}-\tilde{\mu}_{R}\right) \approx \frac{\mu \tan \beta}{m_{3}^{4}}\left[m_{\mu} s_{L}^{2} s_{R}^{2}+\frac{1}{4} m_{\tau} \sin 2 \theta_{L} \sin \theta_{R}\right],
$$

where the second term may dominate. In this case $\delta a_{\mu}$ depends crucially on the mixing angles $\theta_{L}$ and $\theta_{R}$.

The key feature of the effective Lagrangian in Eq. (3) is that there is a chiral flip between the initial and final fermion states. This feature leads to that, in the case of no slepton mixing, all terms in Eqs. (6) and (7) will produce at least one muon mass, $m_{\mu}$, suppression, which either comes from the mass insertion on the external fermion legs, or from the Yukawa coupling vertices, or from the left- and right-handed smuon mixing. This can be explicitly examined by checking all the Feynman diagrams on the interaction basis. The quite interesting point in the case of slepton mixing is that $F\left(\tilde{\mu}_{L}-\tilde{\mu}_{R}\right)$, given in Eq. (28), is approximately proportional to $m_{\tau}$, which can give an enhancement to $\delta a_{\mu}$. This term may dominate others if both the left- and right-handed mixing angles are large. We will show this point in the next section.

From the above analysis we have shown that by changing to the interaction basis, $\delta a_{\mu}$ and $\operatorname{Br}(\tau \rightarrow \mu \gamma)$ can manifest their dependence on the SUSY and mixing parameters. This basis becomes very convenient for our discussion of the relation between $\delta a_{\mu}$ and $\operatorname{Br}(\tau \rightarrow \mu \gamma)$ later. 
It should be mentioned that the parameters $m_{2,3}$ are different for sleptons and sneutrinos, as shown in Eqs. (14,16) and (19). We adopt the same symbol in $F(\tilde{\nu}-\tilde{\nu})$ and $F(\tilde{\mu}-\tilde{\mu})$ only for simplicity. In numerical calculations we adopt the full form in Eqs. (14) and (19).

\section{BOUND ON $\delta a_{\mu}$ AND NUMERICAL RESULTS}

In this section, we focus our discussion on the effective SUSY scenario, i.e., $m_{2} \gg m_{3}$. In this case, $\delta a_{\mu}$ and $\operatorname{Br}(\tau \rightarrow \mu \gamma)$ are closely related, as shown in the last section. We will give an approximate bounds on $\delta a_{\mu}$ through analytic relations between $\delta a_{\mu}$ and $\operatorname{Br}(\tau \rightarrow \mu \gamma)$, taking into account the experimental up limit on the LFV processes. Numerical results are also presented.

The free parameters in this calculation are the Higgsino mass, $\mu, \mathrm{U}(1)_{Y}$ and $\mathrm{SU}(2)_{W}$ gaugino masses, $M_{1}$ and $M_{2}$, ratio of $\mathrm{VEVs}$, $\tan \beta$, mixing angles, $\theta_{L}$ and $\theta_{R}$, slepton mass squares, $m_{\tilde{l}_{2}}^{2}, m_{\tilde{l}_{3}}^{2}, m_{\tilde{r}_{2}}^{2}, m_{\tilde{r}_{3}}^{2}$, and the trilinear terms $A_{l}$. Throughout the whole calculation we fix $A_{l}=0, m_{\tilde{l}_{2}}^{2}=m_{\tilde{r}_{2}}^{2}=20 T e V$. If we do not state explicitly we will take the relation $M_{1}=\frac{5 \alpha_{1}}{3 \alpha_{2}} M_{2}$ and $m_{\tilde{l}_{3}}^{2}=m_{\tilde{r}_{3}}^{2}$. We demand all the SUSY particle spectra be above the present experimental lower limit.

\section{A. $\delta a_{\mu}$ with $\theta_{R}=0$}

When there is only left-handed mixing in the slepton sector, the most important contribution to $\delta a_{\mu}$ and $\operatorname{Br}(\tau \rightarrow \mu \gamma)$ comes from the diagram in FIG. 2, given on the interaction basis. From this diagram we can directly read that

$$
A_{R}^{23}(c) \approx \frac{1}{2} \delta a_{\mu}^{(c)} / m_{\mu}^{2} \frac{F\left(\tilde{\nu}_{\tau}-\tilde{\nu}_{\mu}\right)}{F\left(\tilde{\nu}_{\mu}-\tilde{\nu}_{\mu}\right)} \approx \frac{1}{2} \delta a_{\mu}^{(c)} / m_{\mu}^{2} \frac{c_{L}}{s_{L}}
$$

Then we have, assuming $\theta_{L}=\pi / 4$, that

$$
\begin{aligned}
\operatorname{Br}(\tau \rightarrow \mu \gamma) & \approx \frac{\alpha_{e m}}{4} m_{\tau}^{5}\left|A_{R}^{23}(c)\right|^{2} / \Gamma_{\tau} \\
& \approx \frac{\alpha_{e m}}{4} m_{\tau}^{5} / \Gamma_{\tau}\left|\frac{\delta a_{\mu}^{(c)}}{2 m_{\mu}^{2}} \frac{c_{L}}{s_{L}}\right|^{2} \\
& \approx 2.9 \times 10^{13}\left|\delta a_{\mu}\right|^{2} .
\end{aligned}
$$




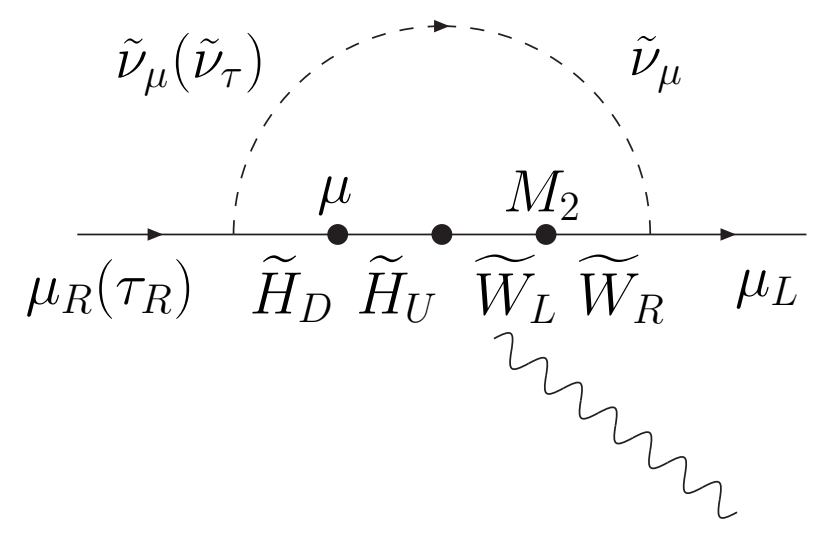

FIG. 2: Feynman diagram which gives the dominant contribution to $\delta a_{\mu}$ (and to the process $\tau \rightarrow \mu \gamma)$ in case of only left-handed slepton mixing. The black dots in the chargino line are mass insertions, with the middle dot representing $\sqrt{2} M_{W} \sin \beta$.

From the present experimental upper bound on $\operatorname{Br}(\tau \rightarrow \mu \gamma)\left(<10^{-6}[\underline{8}]\right)$, we get that

$$
\delta a_{\mu}<1.9 \times 10^{-10}, \text { in case of } \theta_{R}=0 .
$$

From this diagram we also have the conclusion that

$$
\mu M_{2}>0, \text { in case of } \theta_{R}=0
$$

in order that SUSY gives positive contribution to $\delta a_{\mu}$. The same diagram gives the dominant contribution to $\delta a_{\mu}$ in the case of no lepton flavor mixing. Thus the same conclusion of the sign of $\mu$ is given in that case.

Numerical study verifies our above estimation.

B. $\delta a_{\mu}$ with $\theta_{L}=0$

In case of only right-handed mixing, the chargino-sneutrino diagram gives no contribution to $\delta a_{\mu}$. The most important contribution to $\delta a_{\mu}$ and $\operatorname{Br}(\tau \rightarrow \mu \gamma)$ comes from the diagram in FIG. 3, given on the interaction basis. If we ignore the mixing between the left- and 


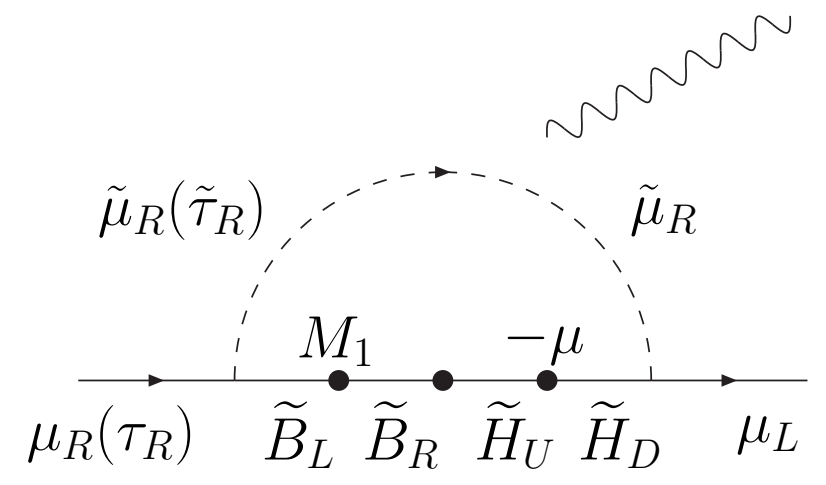

FIG. 3: Feynman diagram which gives the dominant contribution to $\delta a_{\mu}$ (and to the process $\tau \rightarrow \mu \gamma$ ) in case of only right-handed slepton mixing. The black dots in the neutralino line are mass insertions, with the middle dot representing $M_{Z} \sin \beta \sin \theta_{W}$.

right-handed sleptons, $Z_{R}$ is approximately the slepton mixing matrix. From FIG. 3 we then have

$$
A_{R}^{23}(n) \approx \frac{1}{2} \frac{\delta a_{\mu}^{(n)}}{m_{\mu}^{2}}\left(\frac{m_{\mu}}{m_{\tau}}\right) \frac{F\left(\tilde{\tau}_{R}-\tilde{\mu}_{R}\right)}{F\left(\tilde{\mu}_{R}-\tilde{\mu}_{R}\right)} \approx \frac{1}{2} \frac{\delta a_{\mu}^{(n)}}{m_{\mu}^{2}}\left(\frac{m_{\mu}}{m_{\tau}}\right) \frac{c_{R}}{s_{R}} .
$$

Then we have, assuming $\theta_{R}=\pi / 4$, that

$$
\begin{aligned}
\operatorname{Br}(\tau \rightarrow \mu \gamma) & \approx \frac{\alpha_{e m}}{4} m_{\tau}^{5}\left|A_{R}^{23}(n)\right|^{2} / \Gamma_{\tau} \\
& \approx \frac{\alpha_{e m}}{4} m_{\tau}^{5} / \Gamma_{\tau}\left|\frac{\delta a_{\mu}^{(n)}}{2 m_{\mu}^{2}} \frac{m_{\mu}}{m_{\tau}} \frac{c_{R}}{s_{R}}\right|^{2} \\
& \approx 1 . \times 10^{11}\left|\delta a_{\mu}\right|^{2} .
\end{aligned}
$$

From the present upper limit of $\operatorname{Br}(\tau \rightarrow \mu \gamma)<10^{-6}$, we get that

$$
\delta a_{\mu}<32 \times 10^{-10}, \text { in case of } \theta_{L}=0
$$

This upper bound is much larger than that in the case of only left-handed mixing. It is obvious that the factor $\frac{m_{\mu}}{m_{\tau}}$ in Eq. (37), which greatly suppresses $\operatorname{Br}(\tau \rightarrow \mu \gamma)$, helps to increase the bound. This factor comes from the $\mu_{L}-\widetilde{H}_{D}-\tilde{\mu}_{R}$ Yukawa coupling vertex in FIG. [3. where the Higgsino component $\widetilde{H}_{D}$ has to be associated with the muon line since there is only right-handed mixing in the slepton sector. However, in FIG. 2, where the charged Higgsino component $\widetilde{H}_{D}$ is associated with the tau line, no such factor helps to suppress $\operatorname{Br}(\tau \rightarrow \mu \gamma)$. 


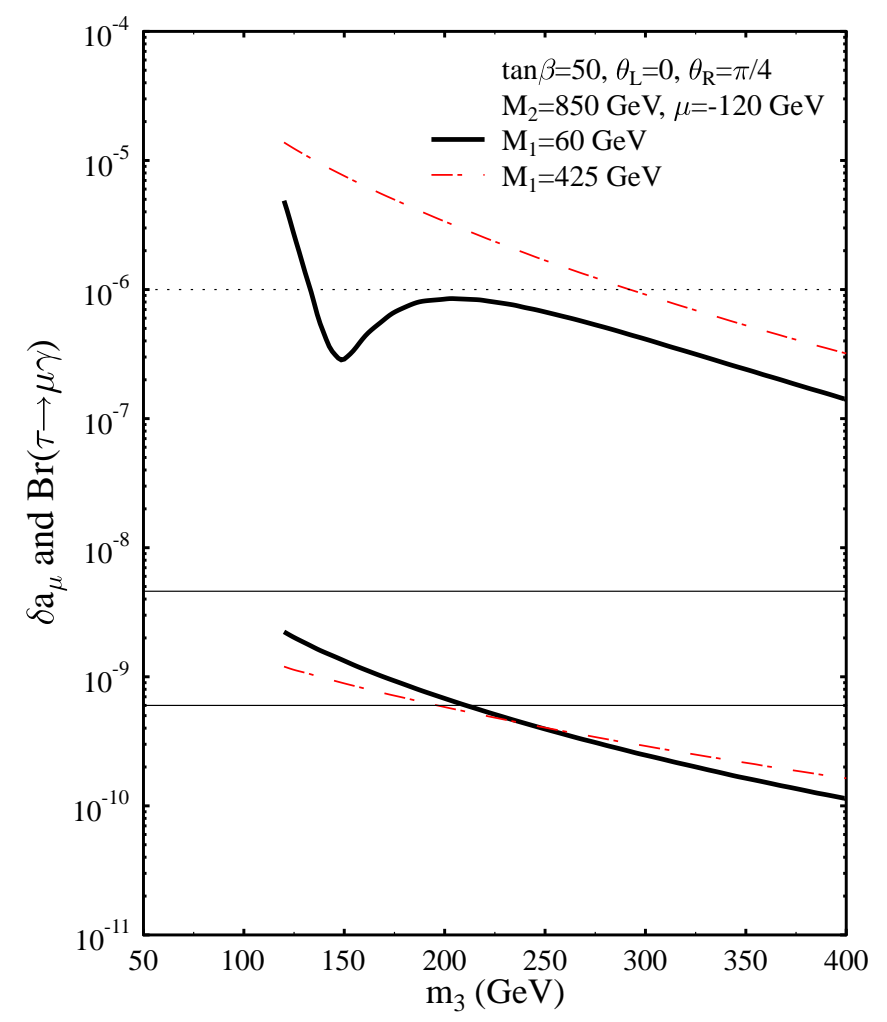

FIG. 4: $\delta a_{\mu}$ and $\operatorname{Br}(\tau \rightarrow \mu \gamma)$ as functions of $m_{3}=m_{\tilde{l}_{3}}=m_{\tilde{r}_{3}}$ in case of $\theta_{L}=0, \theta_{R}=\pi / 4$. The other parameters are $\tan \beta=50, M_{2}=850 \mathrm{GeV} . M_{1}$ is fixed to be $60 \mathrm{GeV}$ for the solid line and $0.5 M_{2}$ for the dashed line. The horizontal lines represent the E821 $\pm 2 \sigma$ bounds (solid) and the upper limit of $\operatorname{Br}(\tau \rightarrow \mu \gamma)$ (dotted).

Another interesting point is that the mass insertion for the neutral component of $\widetilde{H}_{U} \widetilde{H}_{D}$ is $-\mu$, while it is $\mu$ for the same term of the charged component. These terms are clearly shown in the mass matrices of charginos and neutralinos in the Appendix. This sign difference comes in when we contract the SUSY invariant term $\mu \epsilon_{a b} H_{D}^{a} H_{U}^{b}$. Thus we have

$$
\mu M_{1}<0, \text { in case of } \theta_{L}=0
$$

to give positive contribution to $\delta a_{\mu}$. This means that if we set $M_{1}$ and $M_{2}$ have same sign, which is well motivated theoretically, $\mu$ should be negative in this case. We have numerically demonstrated this point by changing the signs of $M_{1}$ and $\mu$ simultaneously and finding that $\delta a_{\mu}$ almost has the same value.

Since we ignored the left-right mixing between the sleptons, the naive bound we get in Eq. (39) should be examined numerically. The numerical results in this case are shown in FIG. 


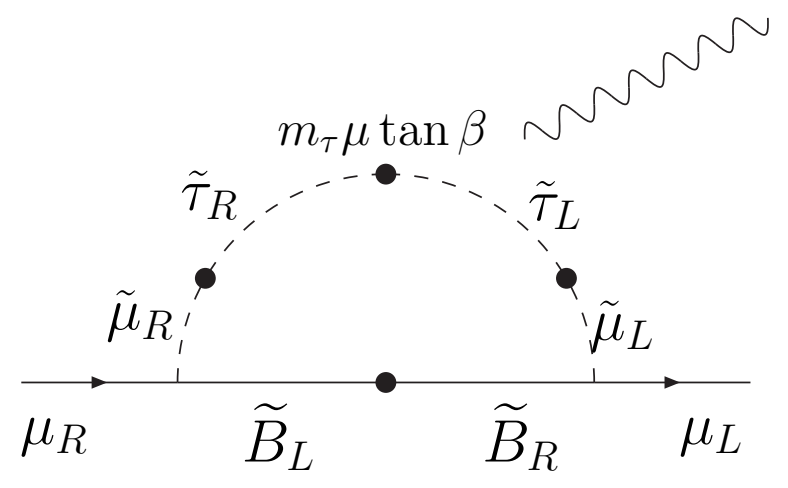

FIG. 5: Feynman diagram which gives the dominant contribution to $\delta a_{\mu}$ in the case that both the left- and right-handed slepton mixing are large.

4. In this figure (and all similar figures below) we draw $\delta a_{\mu}$ and $\operatorname{Br}(\tau \rightarrow \mu \gamma$ ) in the same figure as functions of $m_{\tilde{l}_{3}}=m_{\tilde{r}_{3}}=m_{3}$. The upper group of curves represent $\operatorname{Br}(\tau \rightarrow \mu \gamma)$ while the corresponding curve in the lower group is $\delta a_{\mu}$ with same parameters. The two solid horizontal lines represent the E821 $\pm 2 \sigma$ bounds, $\delta a_{\mu}=6,46 \times 10^{-10}$. The dotted horizontal line is the experimental upper bound on branching ratio of $\tau \rightarrow \mu \gamma, \operatorname{Br}(\tau \rightarrow \mu \gamma)=10^{-6}$.

We take large $\tan \beta(=50)$ and $M_{2}(=850 \mathrm{GeV})$, while $\mu$ is negative. If we adopt the GUT motivated relation $M_{1}=\frac{5 \alpha_{1}}{3 \alpha_{2}} M_{2} \approx 0.5 M_{2}$ we have $\delta a_{\mu}<3 \times 10^{-10}$ to satisfy the $\operatorname{Br}(\tau \rightarrow \mu \gamma)$ bound. However, if we relax the above relation and fix $M_{1}=60 \mathrm{GeV}, \delta a_{\mu}$ can be as large as $\sim 17 \times 10^{-10}$ without violating the bound of $\operatorname{Br}(\tau \rightarrow \mu \gamma)$. This case corresponds to that the LSP (lightest supersymmetric particle) is bino, which is much lighter than other neutralinos.

\section{C. $\delta a_{\mu}$ with no $\theta=0$}

This case is the most general and most interesting one. Our numerical calculation mainly focus on this case. In this case we have derived in Eq. (28) that there is an $m_{\tau}$ enhancement to $F\left(\tilde{\mu}_{L}-\tilde{\mu}_{R}\right)$ if both the left- and right-handed mixing is large in the slepton sector. The enhancement leads to that the diagram in FIG. 5 may give dominant contribution to $\delta a_{\mu}$ if both $\theta_{L}$ and $\theta_{R}$ are large. However, there is no obvious term which give dominant contribution to $\operatorname{Br}(\tau \rightarrow \mu \gamma)$. We find that in small $m_{3}$ region the diagram in FIG. 5 with 


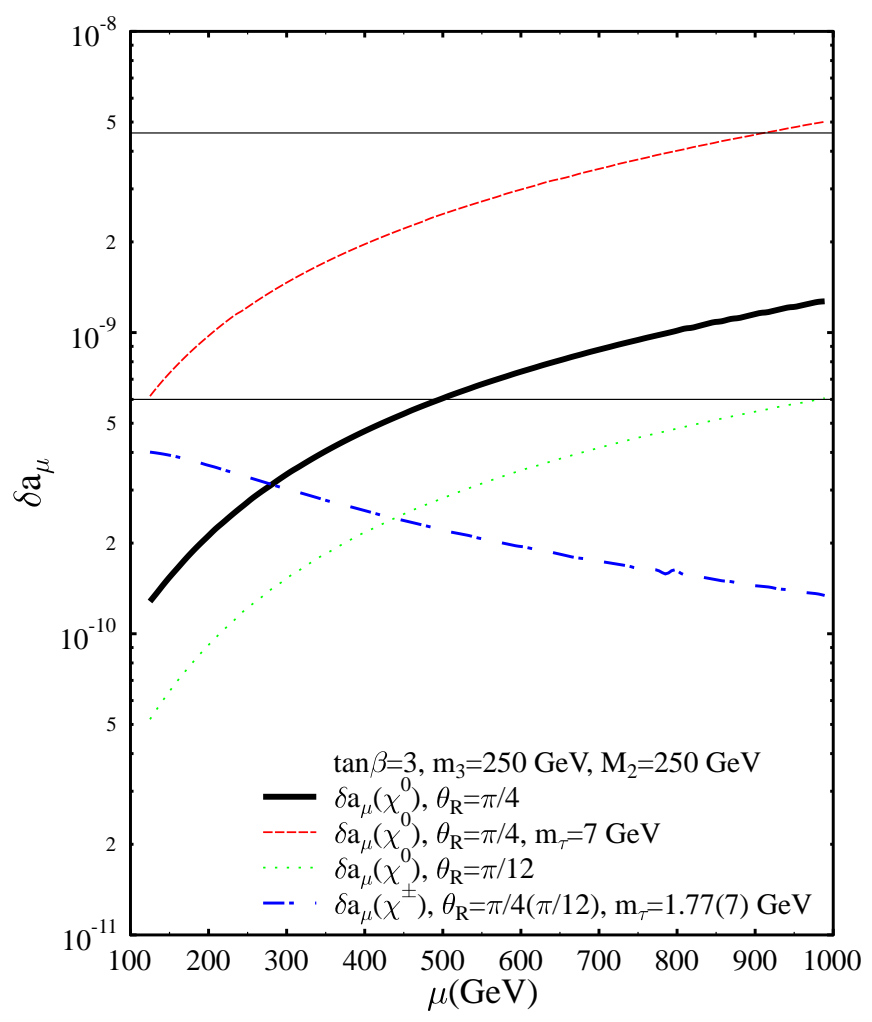

FIG. 6: $\delta a_{\mu}$ as function of $\mu$ for $\tan \beta=3, M_{2}=250 \mathrm{GeV}, m_{3}=250 \mathrm{GeV}$ and $\theta_{L}=\pi / 4$. $\delta a_{\mu}\left(\chi^{0}\right)$ and $\delta a_{\mu}\left(\chi^{ \pm}\right)$represent the contribution coming from exchanging neutralino and chargino respectively. The horizontal lines represent the E $821 \pm 2 \sigma$ bounds of $\delta a_{\mu}$.

$\mu_{R}$ replaced by $\tau_{R}$ may dominates other terms to $\operatorname{Br}(\tau \rightarrow \mu \gamma)$. In this case we get a similar limit as that given in the case with only right-handed mixing, i.e.,

$$
\delta a_{\mu}<32 \times 10^{-10} \text { in case of no } \theta=0
$$

However, this bound is very loose because in large parameter space the contribution to $\operatorname{Br}(\tau \rightarrow \mu \gamma)$ by exchanging $\chi^{ \pm}$is more important than that by exchanging $\chi^{0}$. We can not get simple relation between $\delta a_{\mu}$ and $\operatorname{Br}(\tau \rightarrow \mu \gamma)$ for this case and have to study it numerically.

At first we will numerically verify that FIG. 5 indeed gives important contribution to $\delta a_{\mu}$ by displaying the contributions from exchanging $\chi^{0}$ and $\chi^{ \pm}$separately in FIG. 6. In order to show the $m_{\tau}$ enhancement and the dependence on the mixing angles, we plot another two lines for setting $m_{\tau}=7 G e V$ in the slepton mass matrix and for $\theta_{R}=\pi / 12$. We notice that $\delta a_{\mu}$ changes linearly as $m_{\tau}$, demonstrating the term proportional to $m_{\tau}$ indeed gives 


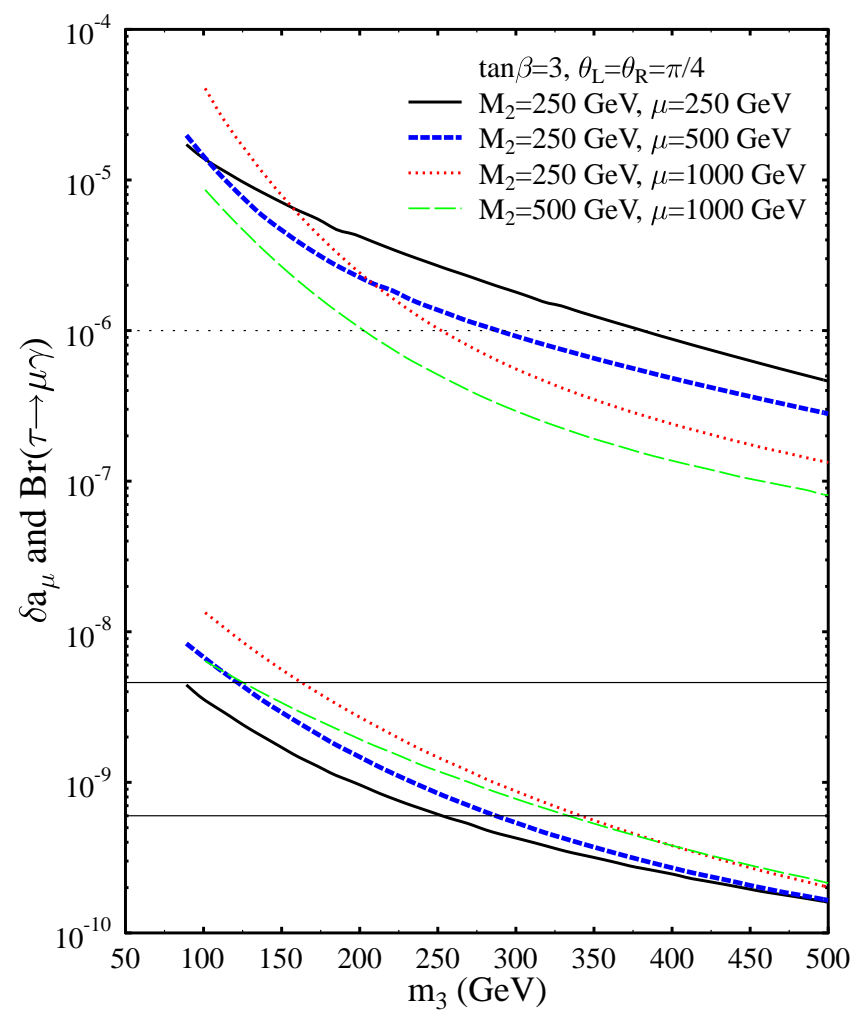

FIG. 7: $\delta a_{\mu}$ and $\operatorname{Br}(\tau \rightarrow \mu \gamma)$ as functions of $m_{3}$ for $\theta_{L}=\pi / 4, \theta_{R}=\pi / 4, \tan \beta=3, M_{2}=$ $250,500 \mathrm{GeV}$ and $\mu=250,500,1000 \mathrm{GeV}$. The horizontal lines represent the $\mathrm{E} 821 \pm 2 \sigma$ bounds of $\delta a_{\mu}$ (solid) and the upper limit of $\operatorname{Br}(\tau \rightarrow \mu \gamma)$ (dotted).

dominant contribution to $\delta a_{\mu}$. However, contribution from exchanging charginos has no change by changing values of $m_{\tau}$ and $\theta_{R}$. From Eq. (28) it is obvious that the neutralino contribution becomes large as $\mu$ increases, while the chargino contribution becomes small since large $\mu$ leads to heavy chargino mass.

FIG. [7 displays $\delta a_{\mu}$ and $\operatorname{Br}(\tau \rightarrow \mu \gamma)$ for $\tan \beta=3, \theta_{L}=\theta_{R}=\pi / 4$. If both $M_{2}$ and $\mu$ are large, there is a large region which can accommodate $\delta a_{\mu}$ and $\operatorname{Br}(\tau \rightarrow \mu \gamma)$ simultaneously. As $\mu$ becomes large, $\operatorname{Br}(\tau \rightarrow \mu \gamma)$ decreases while $\delta a_{\mu}$ increases. This is understood that large $\mu$ enhances $F\left(\tilde{\mu}_{L}-\tilde{\mu}_{R}\right)$ and leads to large chargino mass, which decreases $\operatorname{Br}(\tau \rightarrow \mu \gamma)$.

In FIG. 8 we relax the relation $m_{\tilde{l}_{3}}=m_{\tilde{r}_{3}}$. We show $\delta a_{\mu}$ and $\operatorname{Br}(\tau \rightarrow \mu \gamma)$ as functions of $m_{\tilde{l}_{3}}=m_{3}$ for $m_{\tilde{r}_{3}}=1.5 m_{3}$ and $m_{\tilde{r}_{3}}=220 \mathrm{GeV}$. From Eq. (28) we notice that the sign of $\mu$ can be either positive or negative depending on the relative sign of $\theta_{L}$ and $\theta_{R}$. We also plot $\delta a_{\mu}$ and $\operatorname{Br}(\tau \rightarrow \mu \gamma)$ for changing the sign of $\mu$ and $\theta_{R}$ simultaneously in FIG. 8. There is 


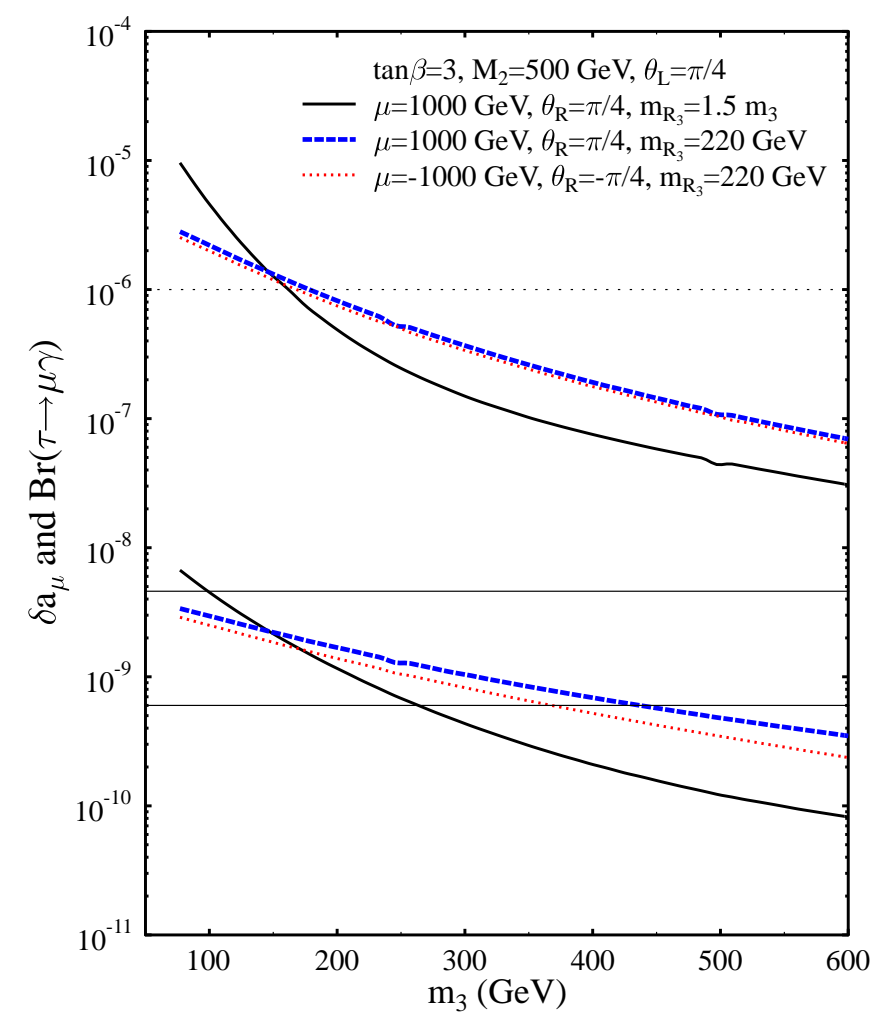

FIG. 8: $\delta a_{\mu}$ and $\operatorname{Br}(\tau \rightarrow \mu \gamma)$ as functions of $m_{\tilde{l}_{3}}=m_{3}$ for $\tan \beta=3, M_{2}=500 G e V$ and $\theta_{L}=\pi / 4$. Taking $m_{\tilde{r}_{3}}=1.5 m_{3}, 220 \mathrm{GeV}, \mu= \pm 1000 \mathrm{GeV}$ and $\theta_{R}= \pm \pi / 4$ respectively. The horizontal lines represent the $\mathrm{E} 821 \pm 2 \sigma$ bounds of $\delta a_{\mu}$ (solid) and the upper limit of $\operatorname{Br}(\tau \rightarrow \mu \gamma)$ (dotted).

little effect on $\delta a_{\mu}$ by the sign reverse.

Since $\operatorname{Br}(\tau \rightarrow \mu \gamma)$ is approximately proportional to $\tan ^{2} \beta$, its upper limit constrains $\tan \beta$ strongly. In above figures we take $\tan \beta=3$. In FIG. 9 we plot $\delta a_{\mu}$ and $\operatorname{Br}(\tau \rightarrow \mu \gamma)$ as functions of $m_{\tilde{l}_{3}}=m_{3}$ for $\tan \beta=10$, and $\mu=1000 \mathrm{GeV}, M_{2}=400,800 \mathrm{GeV}, M_{1}=$ $\frac{5 \alpha_{1}}{3 \alpha_{2}} \approx 0.5 M_{2}, 60 \mathrm{GeV}$ respectively. We fix $m_{\tilde{r}_{3}}=300 \mathrm{GeV}$ in this figure. We can see that when $\tan \beta$ is as large as 10 there is still a large region for $m_{3}$ to accommodate $\delta a_{\mu}$ and $\operatorname{Br}(\tau \rightarrow \mu \gamma)$ simultaneously if $M_{2}$ is large. Generally $m_{3}$ takes larger value than that in case of $\tan \beta=3$ to satisfy the $\delta a_{\mu}$ and $\operatorname{Br}(\tau \rightarrow \mu \gamma)$ bounds. We also notice that relaxing the relation $M_{1}=0.5 M_{2}$ does not change the result much.

In summary, in the effective SUSY scenario, when both the left- and right-handed slepton mixing is large, SUSY can enhance $\delta a_{\mu}$ to within the E821 $\pm 2 \sigma$ bounds in a large parameter space, which can satisfy the constraints by experimental limit on $\operatorname{Br}(\tau \rightarrow \mu \gamma)$. In this 


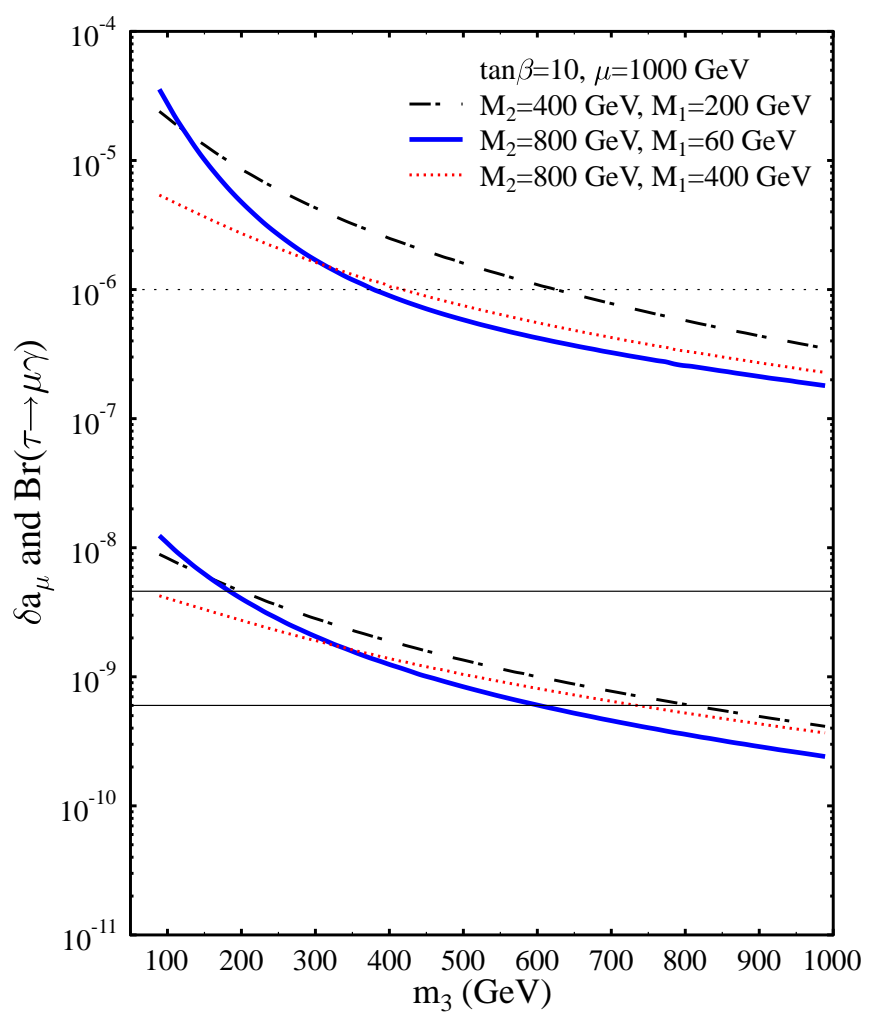

FIG. 9: $\delta a_{\mu}$ and $\operatorname{Br}(\tau \rightarrow \mu \gamma)$ as functions of $m_{\tilde{l}_{3}}=m_{3}$ for $\tan \beta=10, \mu=1000 \mathrm{GeV}, \theta_{L}=\theta_{R}=$ $\pi / 4, m_{\tilde{r}_{3}}=300 \mathrm{GeV}, M_{2}=400,800 \mathrm{GeV}$ and $M_{1}=0.5 M_{2}, 60 \mathrm{GeV}$. The horizontal lines represent the E821 $\pm 2 \sigma$ bounds of $\delta a_{\mu}$ (solid) and the upper limit of $\operatorname{Br}(\tau \rightarrow \mu \gamma)$ (dotted).

case small $\tan \beta$ is slightly favored. Higgsino mass parameter $\mu$ can be either positive or negative depending on the relative sign between $\theta_{L}$ and $\theta_{R}$. We find that $\delta a_{\mu}$ can reach up to $\sim 20 \times 10^{-10}$ even keeping the relation $M_{1} \approx 0.5 M_{2}$, implying that bino is not necessarily kept very light in this case.

\section{SUMMARY AND CONCLUSIONS}

In this work we study the correlation between the SUSY contribution to $\delta a_{\mu}$ and $\operatorname{Br}(\tau \rightarrow$ $\mu \gamma$ ) by translating the analytic expressions in the mass eigenstates to interaction basis, where the gauge coupling and Yukawa interaction vertices are all diagonal. If the slepton mass eigenstates are approximately degenerate, $\delta a_{\mu}$ does not depend on the lepton flavor mixing angles and has no direct relation with the LFV processes. In this case, the analysis 
of $\delta a_{\mu}$ is actually the same as no slepton mixing, as most authors done in the literature. Another case that the second (and first) generation slepton is much heavier than the third generation slepton corresponds to the scenario of effective SUSY. We mainly investigate this case in our work.

In the effective SUSY scenario, if there is only left-handed mixing on the slepton sector, the upper limit of $\operatorname{Br}(\tau \rightarrow \mu \gamma)$ constrains SUSY contribution to $a_{\mu}$ smaller than $1.9 \times 10^{-10}$. In the case of only right-handed mixing on the slepton sector, numerical study shows that $\delta a_{\mu}$ can be at most $\sim 17 \times 10^{-10}$ if bino is the LSP and much lighter than other neutralinos. In this case the Higgsino mass $\mu$ is negative relative to $M_{1}$ in order to give positive contribution to $a_{\mu}$. In the case of both left- and right-handed mixing angles being large, we find the diagram exchanging bino can give dominant contribution to $\delta a_{\mu}$. The sign of $\mu$ is determined by making this diagram positive. Thus $\mu$ can be either positive or negative depending on the relative sign between the left- and right-handed slepton mixing angles. Numerical study shows that in this case there is large parameter space accommodating $\delta a_{\mu}$ and $\operatorname{Br}(\tau \rightarrow \mu \gamma)$ simultaneously. The SUSY contribution to $a_{\mu}$ can reach up to $\sim 20 \times 10^{-10}$, without requiring a very light bino.

Our study shows that the parameter space is quite different in the effective SUSY scenario compared with that in the case of no slepton mixing. The small $\tan \beta$ value is more favored. The sign of $\mu$ is not constrained by the $\left(g_{\mu}-2\right)$ experiment. Finally the effective SUSY scenario can not be excluded by the E821 experiment if we take the lepton flavor mixing effects into account.

\section{APPENDIX}

In this appendix we present our conventions for the SUSY parameters and some analytic expressions for $\delta a_{\mu}$ and $\operatorname{Br}(\tau \rightarrow \mu \gamma)$. For most part we adopt the conventions given in Ref. 9].

The 2-component charged Higgsinos, $\widetilde{H}_{D}^{2}, \widetilde{H}_{U}^{1}$, and charged winos, $\lambda^{ \pm}=\frac{1}{\sqrt{2}}\left(\lambda_{W}^{1} \mp i \lambda_{W}^{2}\right)$, combine to give two 4-component Dirac fermions named charginos, where $\widetilde{H}_{D}^{2}$ and $\widetilde{H}_{U}^{1}$ are the second and the first components of the down and up Higgsino SU(2) doublets respectively, $\lambda_{W}^{1}$ and $\lambda_{W}^{2}$ are the first and the second components of the wino $\mathrm{SU}(2)$ triplet. The mass 
matrix of charginos, given on the interaction eigenstates, is

$$
M_{\chi}=\left[\begin{array}{cc}
m_{2} & \sqrt{2} M_{W} \sin \beta \\
\sqrt{2} M_{W} \cos \beta & \mu
\end{array}\right] .
$$

The unitary mixing matrices $Z^{-}, Z^{+}$satisfy

$$
\left(Z^{-}\right)^{T} M_{\chi} Z^{+}=\operatorname{diag}\left(m_{\chi_{1}}, m_{\chi_{2}}\right)
$$

which are defined by

$$
\left(\begin{array}{c}
-i \lambda^{-} \\
\widetilde{H}_{D}^{2}
\end{array}\right)=Z^{-}\left(\begin{array}{c}
\varphi_{1}^{-} \\
\varphi_{2}^{-}
\end{array}\right)
$$

and

$$
\left(\begin{array}{c}
-i \lambda^{+} \\
\widetilde{H}_{U}^{1}
\end{array}\right)=Z^{+}\left(\begin{array}{c}
\varphi_{1}^{+} \\
\varphi_{2}^{+}
\end{array}\right)
$$

The four-component Dirac charginos are defined by $\chi_{i}^{+}=\left[\begin{array}{c}\varphi_{i}^{+} \\ -\varphi_{i}^{-}\end{array}\right]$. The mass term which will appear in the final form of Lagrangian is $-m_{\chi_{i}} \bar{\chi}_{i} \chi_{i}$.

The third component of wino, $\lambda_{W}^{3}$, bino, $\tilde{B}$, and neutral Higgsinos, $\widetilde{H}_{D}^{1}, \widetilde{H}_{U}^{2}$, combine to give four Majorana neutralinos. The mass matrix for neutralinos on the interaction basis is

$$
M_{\chi^{0}}=\left[\begin{array}{cccc}
m_{1} & 0 & -M_{Z} \cos \beta \sin \theta_{W} & M_{Z} \sin \beta \sin \theta_{W} \\
0 & m_{2} & M_{Z} \cos \beta \cos \theta_{W} & -M_{Z} \sin \beta \cos \theta_{W} \\
-M_{Z} \cos \beta \sin \theta_{W} & M_{Z} \cos \beta \cos \theta_{W} & 0 & -\mu \\
M_{Z} \sin \beta \sin \theta_{W} & -M_{Z} \sin \beta \cos \theta_{W} & -\mu & 0
\end{array}\right],
$$

which is diagonalized by

$$
Z_{N}^{T} M_{\chi^{0}} Z_{N}=\operatorname{diag}\left(m_{\chi_{1}^{0}}, m_{\chi_{2}^{0}}, m_{\chi_{3}^{0}}, m_{\chi_{4}^{0}}\right)
$$

The unitary mixing matrix $Z_{N}$ is defined by

$$
\left(\begin{array}{c}
-i \lambda_{B} \\
-i \lambda_{W}^{3} \\
\widetilde{H}_{D}^{1} \\
\widetilde{H}_{U}^{2}
\end{array}\right)=Z_{N}\left(\begin{array}{c}
\varphi_{1}^{0} \\
\varphi_{2}^{0} \\
\varphi_{3}^{0} \\
\varphi_{4}^{0}
\end{array}\right)
$$


The four-component Majorana neutralinos are given by $\chi_{i}^{0}=\left[\begin{array}{c}\varphi_{i}^{0} \\ \overline{\varphi_{i}^{0}}\end{array}\right]$. The mass term in the final form of Lagrangian of neutralino is $-\frac{1}{2} m_{\chi_{i}^{0}} \bar{\chi}_{i}^{0} \chi_{i}^{0}$.

The mass matrices for sleptons and sneutrinos have been given in Eqs. (14) and (19). They are diagonalized by

$$
Z_{\tilde{L}}^{\dagger} M_{\tilde{l}}^{2} Z_{\tilde{L}}=\operatorname{diag}\left(m_{\tilde{l}_{\alpha}}^{2}\right), \alpha=1 \cdots 6,
$$

and

$$
Z_{\tilde{\nu}}^{\dagger} M_{\tilde{\nu}}^{2} Z_{\tilde{\nu}}=\operatorname{diag}\left(m_{\tilde{\nu}_{\alpha}}^{2}\right), \quad \alpha=1,2,3
$$

The relation between the gauge eigenstates and the mass eigenstates are (omitting the first generation)

$$
\left(\begin{array}{c}
\tilde{\mu}_{L} \\
\tilde{\tau}_{L} \\
\tilde{\mu}_{R}^{*} \\
\tilde{\tau}_{R}^{*}
\end{array}\right)=Z_{\widetilde{L}}\left(\begin{array}{c}
m_{\tilde{l}_{1}}^{2} \\
m_{\tilde{l}_{2}}^{2} \\
m_{\tilde{l}_{3}}^{2} \\
m_{\tilde{l}_{4}}^{2}
\end{array}\right)
$$

and similar expression for sneutrinos.

The functions $F_{i}(k)$ in Eqs. (6) $-(17)$ and (10) -(13) are given as follows. For neutralinoexchange we have

$$
\begin{aligned}
& F_{1}(k)=\frac{1-6 k+3 k^{2}+2 k^{3}-6 k^{2} \log k}{6(1-k)^{4}}, \\
& F_{2}(k)=\frac{1-k^{2}+2 k \log k}{(1-k)^{3}},
\end{aligned}
$$

with $k_{\alpha a}=m_{\chi_{a}^{0}}^{2} / m_{\tilde{l}_{\alpha}}^{2}$. For chargino-exchange we have

$$
\begin{aligned}
& F_{3}(k)=\frac{2+3 k-6 k^{2}+k^{3}+6 k \log k}{6(1-k)^{4}} \\
& F_{4}(k)=\frac{3-4 k+k^{2}+2 \log k}{(1-k)^{3}}
\end{aligned}
$$

with $k_{\alpha a}=m_{\chi_{a}^{-}}^{2} / m_{\tilde{\nu}_{\alpha}}^{2}$. 


\section{ACKNOWLEDGMENTS}

This work is supported by the National Natural Science Foundation of China under the grant No. 10105004.

[1] G. W.Bennet et al., Muon g-2 Collaboration, Phys. Rev. Lett. 89, 101804 (2002); Erratum-ibid. 89, 129903 (2002), arXiv: hep-ex/0208001.

[2] H. N. Brown et al., Muon g-2 Collaboration Phys. Rev. Lett. 86, 2227 (2001).

[3] L. L. Everett, G. L. Kane, S. Rigolin and L. T. Wang, Phys. Rev. Lett. 86, 3484 (2001); J. L. Feng and K. T. Matchev, Phys. Rev. Lett. 86, 3480 (2001); E. A. Baltz and P. Gondolo, Phys. Rev. Lett. 86, 5004 (2001); U. Chattopadhyay and P. Nath, Phys. Rev. Lett. 86, 5854 (2001); R. Arnowitt, B. Dutta, B. Hu and Y. Santoso, Phys. Lett. B505, 177 (2001); S. Komine, T. Moroi and M. Yamaguchi, Phys. Lett. B506, 93 (2001); J. R. Ellis, D. V. Nanopoulos and K. A. Olive, Phys. Lett. B508, 65 (2001); J. Hisano and K. Tobe, Phys. Lett. B510, 197 (2001); Z. Chacko and Graham D. Kribs, Phys. Rev. D 64075015 (2001); K. Choi, K. Hwang, S. K. Kang, K. Y. Lee and W. Y. Song, Phys. Rev. D 64, 055001 (2001); S. P. Martin and J. D. Wells, Phys. Rev. D 64, 035003 (2001); S. Komine, T. Moroi and M. Yamaguchi, Phys. Lett. B507, 224 (2001); S. w. Baek, P. Ko and H. S. Lee, Phys. Rev. D 65, 035004 (2002); D. F. Carvalho, J. R. Ellis, M. E. Gomez and S. Lola, Phys. Lett. B515, 323 (2001); H. Baer, C. Balazs, J. Ferrandis and X. Tata, Phys. Rev. D 64, 035004 (2001); S. w. Baek, T. Goto, Y. Okada and K. i. Okumura, Phys. Rev. D 64, 095001 (2001); G. C. Cho and K. Hagiwara, Phys. Lett. B514, 123 (2001); E. A. Baltz, P. Gondolo, arXiv:astro-ph/0207673 U. Chattopadhyay, P. Nath, arXiv:hep-ph/0208012 M. Byrne, C. Kolda, J. E. Lennon, arXiv:hep-ph/0208067.

[4] S. Baek, P. Ko and J. H. Park, Eur. Phys. J. C24 613 (2002), arXiv:hep-ph/0203251 G. C. Cho, N. Haba and J. Hisano, Phys. Lett. B529, 117 (2002), arXiv:hep-ph/0112163.

[5] K. Hagiwara et al., Phys. Rev. D 66, 01001 (2002).

[6] X. J. Bi and Y. B. Dai, Phys. Rev. D 66, 076006 (2002).

[7] M. Dine, A. Kagan and S. Samuel, Phys. Lett. B243, 250 (1990) ; S. Dimopoulos and G. F. Giudice, Phys. Lett. B357, 573 (1995); A. Pomarol and D. Tommasini, Nucl. Phys. B466, 3 
(1996); A. G. Cohen, D. B. Kaplan and A. E. Nelson, Phys. Lett. B388, 588 (1996); D. E. Kaplan, F. Lepeintre, A. Masiero, A. E. Nelson and A. Riotto, Phys. Rev. D 60, 055003 (1999); J. Hisano, K. Kurosawa and Y. Nomura, Phys. Lett. B445, 316 (1999); J. Hisano, K. Kurosawa and Y. Nomura, Nucl. Phys. B584, 3 (2000).

[8] A. Abe et al., BELLE Collaboration, BELLE-CONF-0118.

[9] J. Rosiek, Phys. Rev. D. 41, 3464 (1990), arXiv: hep-ph/9511250 